\title{
CAUSAS DE VARIAÇÕES NÃO GENÉTICAS E INTERAÇÕES ESTAÇÃO X REGIÃO EM PESOS DE ANIMAIS DE REBANHOS NELORE MOCHO EM ÁREAS INCLUSAS NA REGIÃO PECUÁRIA DE LEITEIRAS
}

\section{(Causes of non-genetic variation and interaction station $\mathrm{x}$ region on weight of cattle animals of Nelore Polled in areas within milk cattle raising region)}

\author{
PAYÁ, S.B. ${ }^{1}$; SIMÕES, A.C.1; FERRAZ FILHO, P.B. ${ }^{2}$; SILVA, L.O.C. ${ }^{3}$; SOUZA, J.C. ${ }^{4}$ \\ 1 Bolsista PIBIC/UFMS/CNPq. Departamento de Ciências Biológicas e da Saúde / \\ UFMS-DCN/UFMS, - paulo@ceul.ufms.br \\ 3 Pesquisador Embrapa Gado de Corte, locs@cnpgc.embrapa.br \\ 4 DZO/UFPR. - jcs@ufpr.br
}

\begin{abstract}
RESUMO - Estudou-se os efeitos de fatores não genéticos sobre 10289, 7981 e 5792 observações de pesos a desmama (ajustado para 205 dias P205), ao ano (365 dias - P365) e sobreano (550 dias - P550), para rebanhos criados em duas sub áreas, Campinas e Ribeirão Preto, inclusas na região pecuária Leiteiras. O modelo estatístico inclui os efeitos fixos de ano e estação de nascimento, sexo do animal, sub região de criação, interação estação x região, e a covariável idade da vaca ao parto (linear e quadrático); como efeito aleatório, efeito de touro. Os machos foram superiores as fêmeas em $7 \%$ à desmama, $12 \%$ aos 12 meses e $14 \%$ aos 18 meses, com média de peso ajustado de $187,34 \mathrm{Kg}$ (P205), 269,23Kg(P365) e 366,54(P550) para sub região de Campinas; e de $165,01 \mathrm{Kg}$ (P205), 231,48Kg (P365) e 305,33Kg (P550)para sub região de Ribeirão Preto. A interação estação $x$ região revelou maior diferença entre os animais nascidos na estação da seca, em ambas regiões, e em todos os pesos analisados. Os coeficientes, lineares e quadráticos obtidos para a idade da vaca foram: $0,2155822 \mathrm{Kg} \mathrm{e}-0,0010670 \mathrm{Kg}, 0,1600645 \mathrm{e}$ $-0,0008712$ e, 0,1919895 e $-0,0011805$ respectivamente, com pontos de máxima ocorrendo aos 8,4 anos (P205), 7,7 anos (P365) e 6,8 anos (P550) de idade da mãe.
\end{abstract}

PALAVRAS CHAVE: Bovinos de corte; efeitos ambientais; produção animal.

ABSTRACT - A study has been carried about effects of non-genetic factors on 10289, 7981 and 5792 observations of weight at weaning (settled for 205 days - W205), a year (365 - W365) and yearling calf (550 - W550) to cattle raised on two sub-areas (Campinas and Ribeirão Preto) within milk cattle region. The statistic model include the steady effects on the year and season of birth, animal's sex, sub- area's breeding, interaction station $\mathrm{x}$ area, and the covariant cow's age at labor (linear and quadratic), with random effect, bull's effect. The males were higher than females in $7 \%$ at weaning, $12 \%$ at 12 months and $14 \%$ at 18 months, with average weight settled of $187.34 \mathrm{Kg}$ (W205), 269.23Kg (W365), and $366.54 \mathrm{Kg}$ (W550) to the sub-area of Campinas; $165.01 \mathrm{Kg}$ (W205), 231.48Kg (W365) and 305.33Kg (W550) to the sub-area of Ribeirão Preto. The coeficients, liners and quadratic obtained to the cow's age were: $0.2155822 \mathrm{Kg}$ and $-0.0010670 \mathrm{Kg}$, 0.1600645 and -0.0008712 and 0.1919895 and -0.0011805 , with maximum points occuring in 8.4 years (W205), 7.7 years (W365) and 6.8 years (W550) of cow's age.

KEY WORDS: cattle; environmental effects; animal production.

\section{INTRODUÇÃO}

No competitivo mercado pecuário, cada vez mais se faz necessário a utilização de informações quantitativas que permitam a aplicação de métodos modernos de análise genética dos rebanhos. Um melhor conhecimento das causas que influenciam características produtivas, como os pesos corpóreos, possibilitando estimativas em especial de efeitos ambientais, que são de relevante importância para uma melhor atuação e manejo dos animais frente aos fatores que interferem no crescimento/desenvolvimento dos rebanhos bovinos de corte no Brasil.

SOUZA et al. (2003) e TORAL et al. (2004) trabaIhando com animais da raça Nelore no Brasil constataram o efeito da integração genótipo $x$ ambiente. Segundo GEMIN et al. (2004) avaliar e aumentar os conhecimentos de características de desempenho produtivo e reprodutivo dos bovinos de corte por regiões específicas é mais um diferencial com possibilidade 
Causas de variação não genéticas e interações estação $\mathrm{x}$ região em pesos de animais de rebanhos nelore mocho em áreas inclusas na região pecuária de leiteiras

de auxiliar os criadores a aumentar a produtividade do rebanho. SOUZA et al. (2001) e GEMIN et al. (2004) ressaltam a importância de se considerar a influencia dos efeitos de meio bem como a correção para tais efeitos ao se realizar a seleção em bovinos de corte.

Com a disponibilidade de novas tecnologias e o desenvolvimento dos computadores é possível manusear grandes conjuntos de dados utilizando metodologias avançadas e eficientes. Este estudo teve como objetivo identificar a influência das fontes de variações não genéticas sobre os pesos ajustados aos 205, 365 e 550 dias de idade.

\section{MATERIAL E MÉTODO}

Para verificar a influência dos fatores ambientais sobre o desenvolvimento ponderal dos animais, fo- ram analisados registros referentes a informações de 10289, 7981 e 5792, observações de pesos ajustado à desmama (205 dias), ao ano (365 dias) e sobreano (550 dias), de bovinos da raça Nelore Mocha provenientes do Convênio ABCZ/Embrapa Gado de Corte, em fazendas situadas nas sub regiões Campinas e Ribeirão Preto ambas pertencentes à região pecuária Leiteiras (ARRUDA e SUGAI, 1994).

As análises foram conduzidas pelo Método dos Quadrados Mínimos, procedimento GLM do programa "Statistic Analyses System" (SAS, 2006), para identificar os efeitos-não genéticos. O modelo estatístico continha os efeitos fixos de ano e estação de nascimento, sexo, região, a interação estação $x$ região e a covariável de idade da vaca ao parto (linear e quadrático), além do efeito aleatório de touro e do erro, conforme descrito a seguir:

$$
Y_{i j k l m n}=\mu T_{i}+A_{j}+E_{k}+S_{1}+R_{m}+E R_{n}+b_{1}\left(X_{i j k l m n}-\bar{X}\right)+b_{2}\left(X_{i j k l m n}-\bar{X}\right)^{2}+e_{i j k l m n}
$$

em que: $Y_{i j k l m n}=$ variável dependente (P205, P365 e P550); $\mu$ = média geral para as características analisadas; $T_{i}=$ efeito aleatório do reprodutor $\mathrm{i}, A_{j}=$ efeito fixo do ano de nascimento j; $E_{k}=$ efeito fixo da estação k de nascimento; $S_{l}=$ efeito fixo do sexo da cria I; $R_{m}=$ efeito da região $\mathrm{m} ; E x R_{n}=$ interação estação x região; $X_{i j k l m n}=$ idade da vaca ao parto; $b_{1}=$ coeficiente de regressão para idade linear; $b_{2}=$ coeficiente de regressão para idade quadrática; e $e_{\text {hijklmn }}=$ erro aleatório, normal e independentemente distribuído com média zero e variância $\sigma^{2}=1$.

\section{RESULTADOS E DISCUSSÃO}

O peso médio observado e o desvio padrão, no conjunto de ambas as regiões, encontrado para P205 foram de $174,07 \pm 24,18 \mathrm{Kg}$ com coeficiente de variação de 14\%, para P365 foram de 238,06 $\pm 40,33 \mathrm{Kg}$ com coeficiente de variação igual a $17 \%$. Sendo que para o peso aos 550 dias foram observados 320,76 $\pm 56,72 \mathrm{Kg}$ de média com $18 \%$ de coeficiente de variação. As análises de variância e os respectivos níveis de $\alpha$ crítico para a significância estatística do teste F são apresentados na TABELA 1.

TABELA 1 - RESUMO DAS ANÁLISES DE VARIÂNCIA, POR QUADRADOS MÍNIMOS PARA P205, P365 E P550 DE BOVINOS NELORE MOCHO NA REGIÃO PECUÁRIA LEITEIRAS

\begin{tabular}{|c|c|c|c|c|c|c|}
\hline \multirow[b]{2}{*}{$\begin{array}{l}\text { Fonte de } \\
\text { Variação }\end{array}$} & \multicolumn{2}{|c|}{ P205 } & \multicolumn{2}{|c|}{ P365 } & \multicolumn{2}{|c|}{ P550 } \\
\hline & $\begin{array}{l}\text { Graus de } \\
\text { Liberdade }\end{array}$ & $\begin{array}{l}\text { Quadrados } \\
\text { Médios }\end{array}$ & $\begin{array}{l}\text { Graus de } \\
\text { Liberdade }\end{array}$ & $\begin{array}{l}\text { Quadrados } \\
\text { Médios }\end{array}$ & $\begin{array}{l}\text { Graus de } \\
\text { Liberdade }\end{array}$ & $\begin{array}{l}\text { Quadrados } \\
\text { Médios }\end{array}$ \\
\hline Touro & 374 & $2325,08^{* * *}$ & 348 & 5915,08 *** & 312 & $12058,83^{* * *}$ \\
\hline Ano & 24 & 8069,30 *** & 24 & $14771,71^{* * *}$ & 24 & $1615306^{* * *}$ \\
\hline Estação & 1 & 71178,72 *** & 1 & $25153,66^{* * *}$ & 1 & 13389,13 * \\
\hline $\begin{array}{l}\text { Sexo do Ani- } \\
\text { mal }\end{array}$ & 1 & $343997,79 * * *$ & 1 & $1832434,85^{* * *}$ & 1 & $3417875,10 * * *$ \\
\hline $\begin{array}{l}\text { Região de } \\
\text { Criação }\end{array}$ & 1 & 408431,83 *** & 1 & $1125745,14^{* * *}$ & 1 & $1683700,26 * * *$ \\
\hline $\begin{array}{c}\text { Estação x } \\
\text { Região }\end{array}$ & 1 & $8645,28 * * *$ & 1 & 8465,58 * & 1 & 8674,63 ns \\
\hline \multicolumn{7}{|l|}{ Idade da Vaca } \\
\hline Linear & 1 & $38923,25^{* * *}$ & 1 & $16089,95^{* * *}$ & 1 & 17361,69 * \\
\hline Quadrático & 1 & $42440,25^{* * *}$ & 1 & $20960,52 * * *$ & 1 & 28853,88 * \\
\hline Resíduo & 9879 & 584.91 & 7602 & 1626,46 & 5449 & 3217,81 \\
\hline
\end{tabular}

* SIGNIFICANTE A 0,1\%; *** SIGNIFICANTE A 0,0001; NS = NÃO SIGNIFICATIVO. 
Os efeitos fixos de sexo, ano e estação de nascimento e região de criação do animal, além dos efeitos aleatórios de touro foram significativos $(P<0,001)$ em todos os pesos. O efeito linear e quadrático da idade da mãe ao parto também foi significativo $(P<0,001)$ para P205, mas não apresentou o mesmo grau de significância para os pesos pós-desmama (P365 e P550). O mesmo ocorrendo para a influência da interação estação x região.

Com relação ao ano de nascimento, os pesos variaram de $158,54 \pm 2,03 \mathrm{Kg}$ a $191,77 \pm 1,42 \mathrm{Kg}$ aos 205 dias, de $219,94 \pm 3,89 \mathrm{Kg}$ a $265,75 \pm 2,58 \mathrm{Kg}$ aos 365 dias e de $307,21 \pm 8,18 \mathrm{Kg}$ a $363,47 \pm 11,95 \mathrm{Kg}$ aos 550 dias de idade. Estas oscilações provavelmente podem ter sido causadas por diferenças nos manejos nutricional, reprodutivo e sanitário. O efeito da época de nascimento sobre os pesos dos animais reflete as variações das condições temporárias do meio, de uma estação para outra, sobre tudo na disponibilidade de alimentos e no manejo adotado para cria e recria dos animais. Neste estudo foi observado que os animais mais pesados, em todas as idades avaliadas, foram os nascidos na época da seca (maio a setembro) os quais foram superiores em 6Kg (P205), 5Kg (P365) e 4Kg (P550), quando comparados com os nascidos na estação das águas (outubro a abril). Observou-se uma redução na diferença entre as duas estações.

Os machos foram mais pesados $12,15 \mathrm{Kg}(7 \%)$ aos 205 dias, $32,11 \mathrm{Kg}(12 \%)$ aos 365 dias e $52,18 \mathrm{Kg}(15 \%)$ aos 550 dias indicando dimorfismo sexual crescente e concorda com os relatos de FERRAZ FILHO (1996).

As médias ajustadas dos pesos obtidas para a região de Campinas foram 187,34 $\pm 0,94 \mathrm{~kg}$ (P205), $269,23 \pm 1,79 \mathrm{Kg}$ (P365) e 366,54 $\pm 2,04$ (P550), correspondendo, respectivamente, a $39 \%, 56 \%$ e $76 \%$ do peso final de abate $(480 \mathrm{Kg})$, enquanto que os valores médios estimados para Ribeirão Preto foram 165,01 \pm 0,77 Kg (P205), 225,84 $\pm 1,46 \mathrm{Kg}$ (P365) e 305,36 $\pm 2,53 \mathrm{~kg}$ (P550) que equivalem, respectivamente, a $35 \%, 47 \%$ e $64 \%$ do peso terminal. Segundo FERRAZ FILHO (1996), as diferenças entre condições edafoclimáticas, espécies forrageiras, manejos e constituições genéticas, em conjunto ou isoladamente, são fatores que contribuem para as diferenças entre as regiões.

A idade das matrizes quando as progênies apresentaram melhor desempenho (pontos de máxima) foi de 8,4 anos (P205), 7,7 anos (P365) e 6,8 anos (P550) quando as mesmas produziram progênies mais pesadas, concordando com os trabalhos de SOUZA et al. (2000), FERRAZ FILHO et al. (2001) e GARCIA et al. (2003).

Na Tabela 2 são apresentados as médias e errospadrão das características estudadas, de acordo com as interações entre as duas estações de nascimento (seca e águas) e as duas sub Regiões de Criação Campinas (R1) e Ribeirão Preto (R2). Com base no apresentado na Tabela 2 e exposto nas Figura 1, 2 e 3 , pode-se observar que a estação de nascimento 1 , proporcionou os melhores pesos em todas as idades e nas duas regiões.

TABELA 2 - EFEITO DA INTERAÇÃO ESTAÇÃO DE NASCIMENTO E SUB REGIÃO DE CRIAÇÃO SOBRE OS PESOS AOS 205 (P205), 365 (P365) E 550 (P550) DIAS DE IDADE.

\begin{tabular}{lccc}
\hline & \multicolumn{3}{c}{ Médias \pm erros-padrão $(\mathrm{kg})$} \\
\cline { 2 - 4 } Estação x Região & $\mathrm{P} 205$ & $\mathrm{P} 365$ & $\mathrm{P} 550$ \\
\hline E1 x R1 & $189,51 \pm 1,11$ & $270,23 \pm 2,12$ & $366,95 \pm 3,62$ \\
E1 x R2 & $185,17 \pm 1,04$ & $268,24 \pm 2,03$ & $366,13 \pm 3,44$ \\
E2 x R1 & $169,49 \pm 0,84$ & $229,57 \pm 1,58$ & $309,05 \pm 2,72$ \\
E2 x R2 & $160,52 \pm 0,81$ & $222,12 \pm 1,54$ & $301,68 \pm 2,66$ \\
\hline
\end{tabular}

CORRESPONDEM AS ESTAÇÕES SECA (E1) E ÁGUAS (E2), RESPECTIVAMENTE; R1= REGIÃO CAMPINAS E R2 = REGIÃO RIBEIRÃO PRETO.

Verificou-se que os animais nascidos na seca foram mais pesados em todas as idades para as duas regiões. As interações estação x região não apenas mostram a diferença de desempenho entre as duas estações como a mudança em desempenho de um ambiente para o outro, conforme SOUZA et al.(2003). Quando se compara o P205 dentro de região, entre estações, verifica-se uma variação bem maior
$24,7 \mathrm{Kg}(15,4 \%)$ de variação na região de Ribeirão Preto e para a região de Campinas, $20,02 \mathrm{Kg}(11,8 \%$, proporção esta estimada dividindo a diferença entre os pesos pelo menor peso).

Quando se comparou a mesma idade, entre regiões, os animais nascidos na sub região de Campinas apresentaram peso superior, como pode se observar nas figuras 1 a 3 . 
Causas de variação não genéticas e interações estação $\mathrm{x}$ região em pesos de animais de rebanhos nelore mocho em áreas inclusas na região pecuária de leiteiras

FIGURA 1 - EFEITO DA INTERAÇÃO ENTRE A ESTAÇÃO DE NASCIMENTO (1 = SECA, 2 = ÁGUAS) E A REGIÃO DE CRIAÇÃO SOBRE O PESO AOS 205 DIAS DE IDADE.

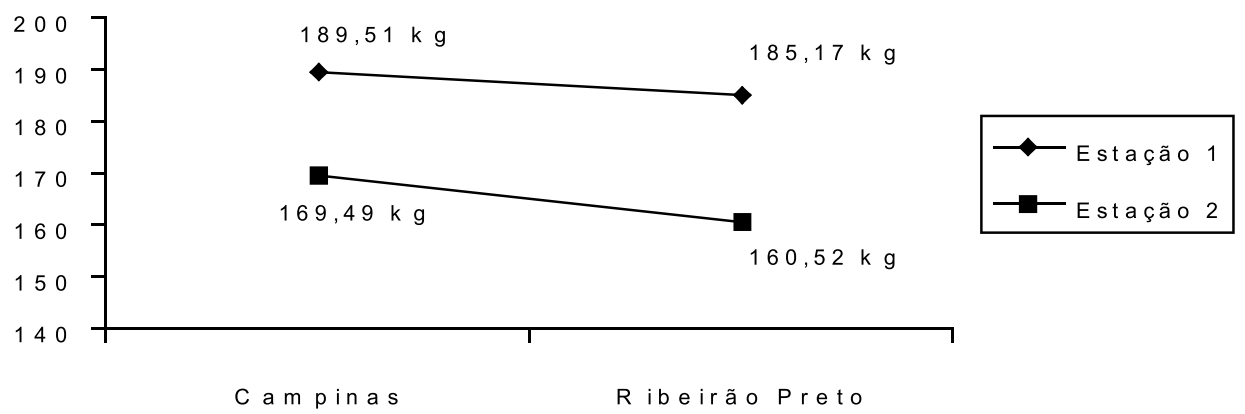

FIGURA 2 - $\quad$ EFEITO DA INTERAÇÃO ENTRE A ESTAÇÃO DE NASCIMENTO IDADE.

( 1 = SECA , 2 = ÁGUAS) E A REGIÃO DE CRIAÇÃO SOBRE O PESO AOS 365 DIAS DE

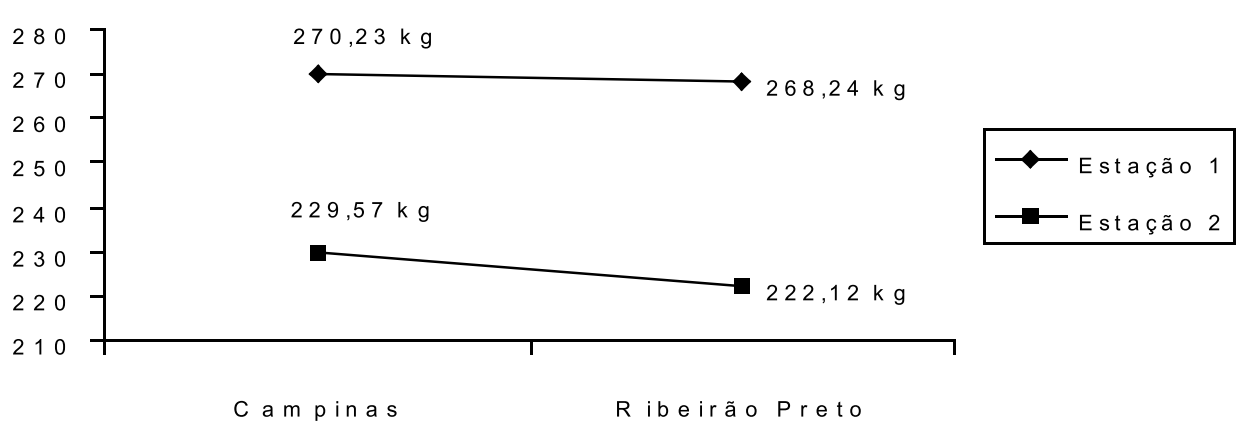

FIGURA 3 - EFEITO DA INTERAÇÃO ENTRE A ESTAÇÃO DE NASCIMENTO IDADE.

( 1 = SECA, 2 = ÁGUAS) E A REGIÃO DE CRIAÇÃO SOBRE O PESO AOS 550 DIAS DE

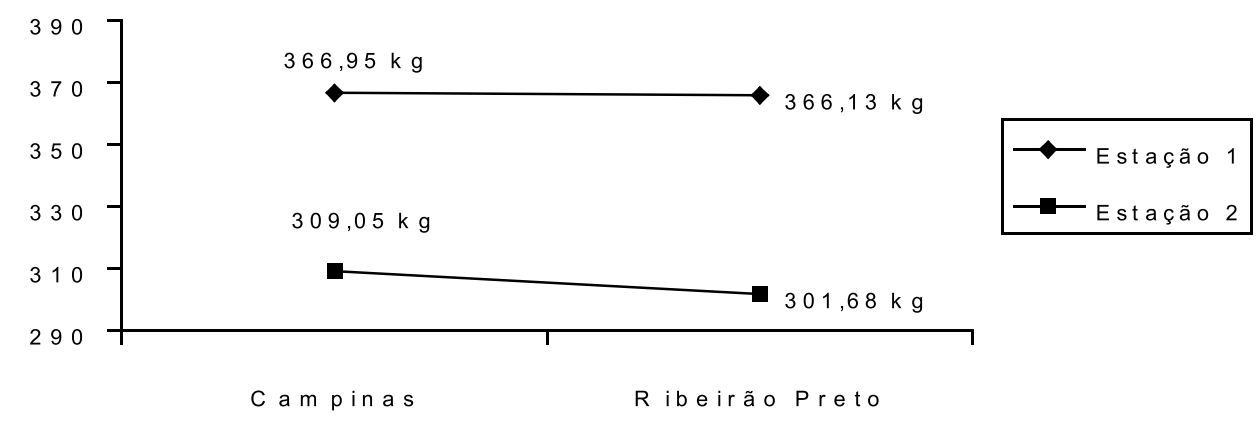

Quanto a variação para o peso aos 365 dias de idade foi menor entre regiões, no entanto, dentro de região entre estação aumentou. Para a sub região de Campinas os animais nascidos na seca foram $17,4 \%$ mais pesados que os nascidos no período das águas e para a sub região de Ribeirão Preto, $20,8 \%$. Finalmente, para o peso aos 550 dias de idade, quase que não houve variação entre sub regiões, mostrando que tal diferença de peso tende a diminuir com o passar da idade, ou seja, os animais chegam ao tamanho adulto com peso similar nas duas regiões, como mostra FERRAZ FILHO (2001). Por outro lado, verificou-se que os animais nascidos na seca forma mais pesados a esta idade que os nascidos no período das águas (Figura 3), com uma superioridade de $18,7 \mathrm{Kg}$ para os animais nascidos na seca na sub região de Campinas e de $21 \%$ para os da sub região de Ribeirão Preto. 
PAYÁ, P. B. F. et al.

\section{CONCLUSÕES}

Constatou-se variação de peso entre e dentro de sub regiões, sendo que os animais nascidos no período da seca foram sempre mais pesados. Os efeitos ambientais influenciaram significativamente as avaliações, devendo sempre fazer as devidas correções.

\section{REFERÊNCIAS}

ARRUDA, Z. J.; SUGAI, Y. Regionalização da pecuária bovina no Brasil. Campo Grande: EMBRAPA-CNPGC; Brasília: EMBRAPA-SPI. 144p - (EMBRAPA-CNPGC, documentos, 58), 1994.

FERRAZ FILHO, P.B. Análise e tendência genética de pesos em bovinos da raça Nelore Mocha no Brasil. Jaboticabal, SP: UNESP, 1996. 163p. Dissertação (Mestrado em Zootecnia) - Universidade Estadual Paulista, 1996.

FERRAZ FILHO, P.B.; RAMOS, A.A.; SILVA, L.O. C.; SOUZA, J.C; ALENCAR, M.M. Environmental and genetic influencing pré and post-weaning growth traits of tabapuã cattle in Brazil. Archives of Veterinary Science, v.6,n.2, p. 19-30, 2001.

FERRAZ FILHO, P.B. Avaliação genética do desenvolvimento ponderal de bovinos da raça Tabapuã no Brasil: Botucatu, SP: UNESP, 2001. 135p. Tese (Doutorado em Genética) - Instituto de Biociências - Universidade Estadual Paulista, 2001.

GARCIA, F.Q., FERRAZ FILHO, P.B, SOUZA, J.C., SILVA, L.O.C. Tendência dos efeitos genéticos diretos e maternos do peso a desmama de bovinos da raça nelore mocha na região pecuária Campo grande e Dourados - Mato Grosso do Sul. Archives of Veterinary Science, v.8, n.1, p. 99-107, 2003.

GEMIN, E.; SOUZA, J.C.; SILVA, L.O.C.; MALHADO, C.H.M.; FERRAZ FILHO, P.B. Efeito do meio e da idade da vaca sobre o ganho de peso na fase de cria, recria e os dias para se obter $160 \mathrm{~kg} \mathrm{em}$ animais da raça Guzerá. Archives of Veterinary Science, v. 9, n. 1, p. 93-99, 2004.

MALHADO, C.H.M., SOUZA, J.C.; SILVA, L.O.C., FERRAZ FILHO, P.B.; WOLF, J.; FREITAS. J.A. Influência da época de nascimento sob as percentagens de crescimento do nascimento aos 550 dias de idade em bovinos da raça Guzerá criados em duas regiões brasileiras. In: XVII Reunión de la Asociación Latino Americana de Produccion Animal, 2001. Anais..., La Habana: ALPA, 2001. v. CD-ROM.

SAS. STATISTICAL ANALYSIS SYSTEM - User Guide: Stat, Version 6.11. Cary, (NC: Sas Institute Inc.) 2006.

SOUZA, J.C.; RAMOS, A.A.; SILVA, L.O.C. EUCLIDES FILHO, K.; ALENCAR, M. M.; VLECK, L.D.V; FERRAZ FILHO, Paulo Bahiense; WECHSLER, Francisco Stefano. Fatores do ambiente sobre o peso ao desmame de bezerros da raça Nelore em regiões tropicais brasileiras. Ciência Rural, v. 30, n. 5, p. 881-885, 2000a.

SOUZA, J.C.; SILVA, L .O.C.; MALHADO, C.H.M., EUCLIDES FILHO, K.; FERRAZ FILHO, P.B; ALENCAR, M.M., FREITAS, J.A. Influência da idade da vaca e correlação genética para pesos de bezerros raça Guzerá, criados nos estados de Minas Gerais e Goiás. In: REUNIÃO ANUAL DA SOCIEDADE BRASILEIRA DE ZOOTECNIA, 37., 2000, Viçosa. Anais... Viçosa: SBZ, 2000b. CD-ROM.

SOUZA, J.C., GADINI, C.H., SILVA, L.O.C., RAMOS, A.A., ALENCAR, M.M., FERRAZ FILHO, P.B. e VAN VLECK, L.D. Estimates of genetic parameters and evaluation of genotype $x$ environment interaction for weaning weight in Nellore cattle. Archivos Latinoamericanos de Producción Animal, v. 11, n. 2, p.94-100, 2003.

TORAL, F.L.B.; SILVA, L.O.C.; MARTINS, E.N. et al. Interação genótipo ambiente em características de crescimento de bovinos de raça Nelore no Mato Grosso do Sul. Revista Brasileira de Zootecnia, v. 33, n. 6, p. 1445-1455, 2004.

WOLF, J., SOUZA, J.C., SILVA, L.O.C., MALHADO, C.H.M., FERRAZ FILHO, P.B. Estudo de efeitos de meio sobre o peso aos 205 e 365 dias em animais da raça Guzerá em três regiões do Brasil. In: XVII Reunión de la Asociación Latino Americana de Produccion Animal, 2001. Anais..., La Habana: ALPA, 2001. v. CD-ROM.

Recebido para publicação em:

$06 / 07 / 2006$ Aprovado:
$04 / 05 / 2007$ 